Relations industrielles

Industrial Relations

\title{
Demain la Santé, Québec, Dossier de Québec-Science, 1976, 291
}

p.

\section{Gilles Dussault}

Volume 31, numéro 3, 1976

URI : https://id.erudit.org/iderudit/028736ar

DOI : https://doi.org/10.7202/028736ar

Aller au sommaire du numéro

Éditeur(s)

Département des relations industrielles de l'Université Laval

ISSN

0034-379X (imprimé)

1703-8138 (numérique)

Découvrir la revue

Citer ce compte rendu

Dussault, G. (1976). Compte rendu de [Demain la Santé, Québec, Dossier de Québec-Science, 1976, 291 p.] Relations industrielles / Industrial Relations, 31(3), 497-498. https://doi.org/10.7202/028736ar

Tous droits réservés @ C Département des relations industrielles de l'Universite Laval, 1976
Ce document est protégé par la loi sur le droit d'auteur. L’utilisation des services d'Érudit (y compris la reproduction) est assujettie à sa politique d'utilisation que vous pouvez consulter en ligne.

https://apropos.erudit.org/fr/usagers/politique-dutilisation/ 
plus que plusieurs modifications ont été apportées à la législation depuis 1970 et que la jurisprudence s'est aussi enrichie.

$\mathrm{Au}$ fait, l'auteur ne s'est pas contenté d'une simple réédition. Utilisant la même méthode, il a revisé complètement son ouvrage et l'a remis à jour en tenant compte de tous les développements juridiques. Aussi on y trouvera une étude des lois suivantes: Loi de la fonction publique, Loi sur les relations du travail dans l'industrie de la construction, Loi sur la mise en tutelle de certains syndicats ouvriers, Loi visant à assurer les services de santé et les services essentiels en cas de conflit de travail. Dans une dernière partie, l'auteur présente une cinquantaine de formules destinées à aider la préparation de demandes, contestations, requêtes et avis au Tribunal du travail, aux cours, administrateurs gouvernementaux, commissaires-enquêteurs, conciliateurs, arbitres et parties à la négociation relativement à l'accréditation, le retrait d'accréditation, la dissolution, les négociations, la conciliation, l'arbitrage, les réclamations pour dommages ou pertes de salaire et les poursuites pénales.

Tous ceux qui œuvrent dans les relations du travail seront reconnaissants à Me Cutler de leur avoir encore une fois fait bénéficier du fruit de sa vaste expérience et de sa compétence reconnue. La seconde édition de son ouvrage indispensable est sûrement appelé à une aussi large diffusion que la première.

Université Laval

Gérard DION

Direction par les objectifs et motivations des hommes par J. D. Batten, Paris, Coll. Gestion, Dalloz, 1976, 89 p.

C'est un ouvrage de moins d'une centaine de pages publié en 1966 sous le titre: «Beyond Management by Objectives.» Il faut se rappeler qu'à ce moment-là, il était plutôt question de gestion par les résultats, sans faire beaucoup de place à une véritable gestion participative par les objectifs impliquant le supérieur hiérarchique et ses collaborateurs immédiats dans la détermination conjointe des objectifs à atteindre dans leur poste respectif et au sein de leur unité administrative. L'ouvrage de Batten se voulait alors une courte réflexion sur une conception améliorée de la gestion par les objectifs cherchant à intégrer la dimension socio-affective et la dimension rationnelle de l'entreprise. En d'autres termes, par delà l'aspect conceptuel et mécanique de ce nouveau système de management, il faut avant tout s'assurer de la motivation des dirigeants à œuvrer dans un tel système; sans cela, les applications les plus élégantes qu'on peut en faire sont vouées à un échec. Pour susciter cette motivation, l'auteur prétend qu'une diffusion adéquate de la philosophie et des objectifs (court terme, long terme) de l'entreprise, après avoir été élaborées à la suite d'une consultation à tous les niveaux, est suffisante pour permettre à tous les cadres de l'entreprise de donner une signification à leurs efforts et à leurs réalisations. C'est une position qui semble au premier abord très acceptable aux U.S.A. puisqu'elle s'intègre parfaitement à une philosophie de la libre entreprise que l'auteur prend soin de rappeler et d'expliciter. Personnellement, je suis convaincu que la dimension motirationnelle est, avant tout, à l'origine du succès de l'implantation et du maintien de ce nouveau système de management. Cependant, elle n'est vraiment assurée que lorsqu'un cadre peut contribuer lui-même à la détermination des objectifs de son poste et de son unité administrative, à l'intérieur du cadre des objectifs et des politiques définies par la hautedirection. En contribuant ainsi à la détermination de ses objectifs de poste, le cadre assume également une responsabilité à l'endroit de résultats à atteindre. En plus de se définir des objectifs de poste, le cadre précise ses objectifs personnels de carrière et de développement. Cette conception véritablement participative de la gestion par les objectifs permet dans une large mesure de concilier la poursuite des objectifs personnels de l'individu avec et ceux de l'organisation qu'il l'emploie. Cette conciliation d'objectifs individuels et organisationnels demeure au cour même de la motivation.

L'ouvrage de Batten propose un type de consultation qui s'inspire de la théorie $\mathrm{Y}$ de McGregor, mais qui avec le recul des années et la poursuite de la réflexion sur le sujet ne nous apparaît plus suffisant nous servir d'assises à la dimension motivationnelle et participative de la gestion par les objectifs.

Université Laval

Laurent BÉLANGER

Demain la Santé, Québec, Dossiers de Québec-Science, 1976, 291 p.

Cet excellent dossier - le premier du genre - que présente le magazine QuébecScience, porte un verdict bien peu reluisant sur le monde de la santé au Québec. L'au- 
teur, Yanick Villedieu a d'ailleurs intitulé son ouvrage Demain la Santé, indiquant par là que la santé reste à conquérir; en effet, jusqu'ici la presque totalité des ressources et énergies ont été utilisées pour lutter contre la maladie et bien peu a été fait pour véritablement promouvoir la santé.

Villedieu a réuni pour ce dossier des études économiques, sociologiques, épidémiologiques et rencontré de nombreux intervenants et observateurs du monde de la santé. Une constante se dégage des études consultées et des commentaires obtenus: la nature sociale de la majeure partie de nos problèmes de santé, que ce soit au niveau de la santé des individus ou à celui de l'organisation institutionnelle. Ce sont les maladies dites de civilisations (maladies ischémiques du cœur, des voies respiratoires, associées directement au stress, au tabagisme, à la mauvaise alimentation, etc...) et des causes reliées à l'environnement ainsi qu'aux comportements individuels (accidents de la route, suicides, alcoolisme, etc...) qui tuent le plus au Québec et qui mobilisent le plus notre système médico-hospitalier. Quant à notre «système de santé», Villedieu montre jusqu'à quel point il est le lieu de nombreuses luttes interprofessionnelles et politiques et combien grande est la distance qui nous sépare d'un système qui promeut la santé plutôt que de s'acharner à contenir les excès d'une société iatrogène. Les dossiers brûlants qu'il met à jour sont avant tout de nature socio-politique: l'épineuse question de la santé au travail en est un exemple. Même si les liens entre conditions de travail et niveaux de santé ont été largement établis, la médecine du travail reste chez nous à peu près inexistante; les législations en vue de la protection de la santé en milieu du travail sont, en général, de très faible portée et le thème même "travail et santé " est encore presque tabou. Car c'est toute l'organisation économique et le système de valeurs qui la sous-tend qui sont rernis en cause lorsqu'on aborde cette question; le moins qu'on puisse dire, c'est qu'il sera de plus en plus difficile d'escamoter le débat sur la santé au travail et qu'on assistera, en cette matière, à de très dures luttes.

Ce dossier fournira aussi des armes à ceux qui comme Ivan Illich pensent que la médecine actuelle, corporatiste, orientée exclusivement vers la distribution de soins, fonctionnant à la manière d'une entreprise capitaliste, est à l'origine de tous nos maux. Il faudrait plutôt souhaiter que ce dossier soit l'amorce d'une réflexion rigoureuse et systématique sur notre façon de combattre la maladie, bien sûr, mais surtout sur les liens qui existent entre notre organisation sociale - notre organisation du travail, en particulier, - les comportements qu'elle génère et les niveaux de santé. Peut-être identifierons-nous alors les éléments fondamentaux d'hygiène, respect de l'environnement, conditions de travail réhumanisées, comportements individuels plus sains, etc..., qui permettront à l'homme moderne de retrouver le véritable «chemin de la santé».

Université Laval

Gilles DUSSAULT

\section{COLLECTION RELATIONS DU TRAVAIL}

Les débrayages massifs de mai 1972 au Québec

Gilles LAFLAMME et Réal ALLARD

département des relations industrielles

Université Laval

Un volume $81 / 2 \times 11,50$ pages $-A$ Book $81 / 2 \times 11,50$ pages

Les Presses de I'Université Laval

Cité Universitaire

Québec, P.Q., Canada

G1K 7R4 